\title{
Numerical Measurements of the Spectrum in Magnetohydrodynamic Turbulence
}

\author{
Joanne Mason, ${ }^{1}$ Fausto Cattaneo, ${ }^{1}$ and Stanislav Boldyrev ${ }^{2}$ \\ ${ }^{1}$ Department of Astronomy and Astrophysics, University of Chicago, 5640 S. Ellis Ave, \\ Chicago, IL 60637; jmason@flash.uchicago.edu; cattaneo@flash.uchicago.edu \\ ${ }^{2}$ Department of Physics, University of Wisconsin at Madison, \\ 1150 University Ave, Madison, WI 53706; boldyrev@wisc.edu
}

(Dated: August 22, 2021)

\begin{abstract}
We report the results of an extensive set of direct numerical simulations of forced, incompressible, magnetohydrodynamic turbulence with a strong guide field. The aim is to resolve the controversy regarding the power law exponent $\left(\alpha\right.$, say) of the field perpendicular energy spectrum $E\left(k_{\perp}\right) \propto k_{\perp}^{\alpha}$. The two main theoretical predictions, $\alpha=-3 / 2$ and $\alpha=-5 / 3$, have both received some support from numerical simulations carried out by different groups. Our simulations have a resolution of $512^{3}$ mesh points, a strong guide field, an anisotropic simulation domain, and implement a broad range of large-scale forcing routines, including those previously reported in the literature. Our findings indicate that the spectrum of well developed, strong incompressible MHD turbulence with a strong guide field is $E\left(k_{\perp}\right) \propto k_{\perp}^{-3 / 2}$.

PACS numbers: 52.30.Cv, 52.35.Ra, 95.30.Qd
\end{abstract}

Introduction.-Magnetohydrodynamic (MHD) turbulence is pervasive in astrophysical systems and plays an important role in stellar interiors, the solar wind and interstellar and intergalactic media (see [1]). Phenomenological models of MHD turbulence aim to describe the dynamics of energy transfer in the spectral domain. They are built upon the foundation that the spatial scales can be separated into three regions: (i) The energy containing range at large scales (small $k$ ) where energy is supplied to the system by an instability or an external force, (ii) the dissipation range at small scales (large $k$ ) where energy is removed from the system by viscosity or resistivity, and (iii) an intermediate region known as the inertial range. Within the inertial range it is assumed that forcing and dissipation are negligible and that energy is transferred from large to small scales solely by nonlinear interactions. It is therefore believed that, regardless of the form of the large-scale energy injection mechanism, once energy has cascaded to sufficiently small scales the nonlinear dynamics are universal (see, e.g., [1] ).

Theoretical predictions for the scaling of the energy spectrum within the inertial range depend upon the assumed physics of the nonlinear interactions. The main theories assume that the basic state of MHD turbulence is one of Alfvén fluctuations: small-scale wave-packets, or eddies, propagate along the large-scale magnetic field with the Alfvén speed $V_{A}=B_{0} / \sqrt{4 \pi \rho}$, where $B_{0}$ is the magnitude of the large-scale field and $\rho$ is the fluid density. Only eddies propagating in opposite directions interact. Iroshnikov [2] and Kraichnan [3] used this fact to develop a theory for three-dimensionally isotropic eddies which need to undergo a large number of interactions to transfer energy to smaller scales. This leads to the Iroshnikov-Kraichnan energy spectrum $E(k) \propto k^{-3 / 2}$.

In later years, observational and numerical evidence revealed that the presence of a strong guiding magnetic field renders the turbulence anisotropic [4, 5, 6, 7, 8]. This motivated Goldreich \& Sridhar [9] to develop a new theory in which the eddies are elongated in the direction of the large-scale field and the energy cascade proceeds mainly in the field perpendicular plane. The eddies are deformed strongly during only one interaction, leading to the field-perpendicular energy spectrum $E\left(k_{\perp}\right) \propto k_{\perp}^{-5 / 3}$.

Recent high resolution numerical simulations verified the anisotropy of the turbulent cascade but also produced the Iroshnikov-Kraichnan exponent for the fieldperpendicular spectrum [10, 11, 12], thus contradicting both the IK and GS models. To address this discrepancy a new theory was presented in 13,14 . In addition to the elongation of the eddies in the direction of the guiding field, therein it is proposed that the fluctuating velocity and magnetic fields at a scale $\lambda \sim 1 / k_{\perp}$ are aligned within a small scale-dependent angle in the field perpendicular plane, $\theta \propto \lambda^{1 / 4}$. The process, known as scale-dependent dynamic alignment, reduces the strength of the nonlinear interactions and leads to the field-perpendicular energy spectrum $E\left(k_{\perp}\right) \propto k_{\perp}^{-3 / 2}$.

Advances in computational power within recent years have made it feasible for a number of independently working groups to test the above predictions via high resolution numerical simulations. The results have led to considerable debate. Although it is largely agreed upon that the turbulent cascade is anisotropic [e.g., 4, 5, 6, 7, 8, 9, 15, 16, 17], and excellent agreement with the scale-dependent alignment $\theta \propto \lambda^{1 / 4}$ has been found [18], there remains issues with regard to the exponent of the field-perpendicular energy spectrum. Some simulations have found $-5 / 3[19,20,21,22$, 23], however, they did not have a strong guide field. Others yield $-3 / 2$, however, either their resolution was limited [10], or the simulation domain was not anisotropic [11, 12], which raised questions whether the field-parallel dynamics were 
captured. In addition, direct comparison of these numerical results is complicated by the fact that each have employed different forcing routines, different dissipation mechanisms, and have different Reynolds numbers.

This principal controversy motivated our research. In an attempt to clarify the above issues, we have conducted a wide range of direct numerical simulations with a resolution of $512^{3}$ mesh points and Reynolds number $R e \approx 2200$. Our simulations have a strong guide field, and an anisotropic simulation box to allow for the field-parallel dynamics. We force wavenumbers $k=1,2$ and we have analyzed 17 different cases in which we vary the relative intensities and correlation times of the forcing for the velocity and magnetic fields. These cases include those settings previously reported in the literature.

In almost all cases our simulations yield the spectrum $-3 / 2$. A steeper spectrum, consistent with $-5 / 3$, is observed in a special case when the velocity field is driven by a force whose correlation time is much shorter than the relaxation time of the large-scale eddies. However, we believe that this is a result of a setting that is not well suited for simulating well-developed strong MHD turbulence, since such short correlation time forcing may spoil an inertial interval of limited extent. In this case we do expect that the universal spectrum $-3 / 2$ should emerge at smaller scales, though a larger calculation with a deeper inertial range is needed to observe it.

A further important finding is that in all cases the scale-dependent dynamic alignment of magnetic and velocity fluctuations, which is thought to be responsible for the $-3 / 2$ spectrum [13, 14, 18, 24], is clearly observed. We therefore conclude that numerical simulations indicate that the spectrum of strong incompressible MHD turbulence with a strong guide field is $E\left(k_{\perp}\right) \propto k_{\perp}^{-3 / 2}$. In this paper we report the main results of our work. A detailed discussion will be presented elsewhere.

Numerical results.-We consider driven incompressible MHD turbulence with a strong guiding magnetic field. The equations read

$$
\begin{gathered}
\partial_{t} \mathbf{v}+(\mathbf{v} \cdot \nabla) \mathbf{v}=-\nabla p+(\nabla \times \mathbf{B}) \times \mathbf{B}+\nu \Delta \mathbf{v}+\mathbf{f}_{\mathbf{v}} \\
\partial_{t} \mathbf{B}=\nabla \times(\mathbf{v} \times \mathbf{B})+\eta \Delta \mathbf{B}+\mathbf{f}_{\mathbf{B}}
\end{gathered}
$$

where $\mathbf{v}(\mathbf{x}, t)$ is the velocity field, $\mathbf{B}(\mathbf{x}, t)$ the magnetic field, $p$ the pressure, and $\nu$ and $\eta$ are the fluid viscosity and resistivity, respectively. They will be solved using standard pseudospectral methods. The external forces $\mathbf{f}_{\mathbf{v}}(\mathbf{x}, t)$ and $\mathbf{f}_{\mathbf{B}}(\mathbf{x}, t)$ drive the turbulence at large scales, though the precise spatial and temporal form of the forcing is free to be chosen. In an attempt to resolve the previously reported controversies, we consider forcing mechanisms in the following three categories (collectively, they contain the main forcing mechanisms used in the recent literature).

Case 1: Random forcing of the velocity field only
$\left(\mathbf{f}_{\mathrm{B}} \equiv 0\right)($ e.g. [22, 23] $)$.

Case 2: Random independent forcing of both the velocity and magnetic fields (e.g. [10]).

Case 3: Steady forcing of both the velocity and magnetic fields by freezing large-scale modes (e.g. [11, 12]).

In cases 1 and 2, our random force satisfies the following requirements: it has no component along $z$, it is solenoidal in the $x-y$ plane, all the Fourier coefficients outside the range $1 \leq k \leq 2$ are zero, the Fourier coefficients inside that range are Gaussian random numbers with variance chosen so that the resulting $\mathrm{rms}$ velocity fluctuations are of order unity. The individual random values are refreshed independently on average at time intervals approximately twice as large as the turnover time of the large-scale eddies. We also consider a special case with a renovation time that is ten times smaller.

In case 3 we initialize the calculation with a constant multiple of the statistically steady state solution from a simulation in which only the velocity is driven. We then evolve only those modes with $k \geq 2$ and we hold fixed the Fourier coefficients of both the velocity and the magnetic fields for those modes in which $k_{x}, k_{y}$ and $k_{z}$ are equal to 0 or \pm 1 (excluding $k_{x}=k_{y}=k_{z}=0$ ). The multiplication factor is chosen so that the solution relaxes to a statistically steady state with $r m s$ velocity fluctuations of order unity.

The following results correspond to simulations that have an external magnetic field applied in the $z$ direction with strength $B_{0} \approx 5$, measured in units of velocity. The periodic domain is elongated in the $z$ direction with aspect ratio 1:1: $B_{0}$. The Reynolds number is defined as $R e=U_{r m s}(L / 2 \pi) / \nu$, where $L(=2 \pi)$ is the field-perpendicular box size, $\nu$ is fluid viscosity, and $U_{r m s}$ $(\sim 1)$ is the rms value of velocity fluctuations. We restrict ourselves to the case in which the magnetic resistivity and fluid viscosity are the same, $\nu=\eta$, with $R e \approx 2200$. The system is evolved until a stationary state is reached (confirmed by observing the time evolution of the total energy of fluctuations). The data sets for each run consist of approximately 30 samples that cover approximately 6 turnover times at the largest scales.

For each simulation we measure the two-dimensional energy spectrum, defined as $E\left(k_{\perp}\right)=\left\langle\left|\mathbf{v}\left(k_{\perp}\right)\right|^{2}\right\rangle k_{\perp}+$ $\left\langle\left|\mathbf{b}\left(k_{\perp}\right)\right|^{2}\right\rangle k_{\perp}$, where $\mathbf{v}\left(k_{\perp}\right)$ and $\mathbf{b}\left(k_{\perp}\right)$ are the twodimensional Fourier transformations of the velocity and magnetic field in a plane perpendicular to $\mathbf{B}_{0}$ and $k_{\perp}=$ $\left(k_{x}^{2}+k_{y}^{2}\right)^{1 / 2}$. The average is taken over all such planes in the data cube, and then over all data cubes.

Shown in Figure 1h is the spectrum of fluctuations for Case 1. To infer whether a $\log -\log$ plot of $E\left(k_{\perp}\right)$ has a slope of $-3 / 2$ or $-5 / 3$ we compensate the spectrum (solid curve) and, for comparison, a function with scaling $k_{\perp}^{-5 / 3}$ (dashed line) by $k_{\perp}^{3 / 2}$. We conclude that the best fit is $E\left(k_{\perp}\right) \propto k_{\perp}^{-3 / 2}$, with the inertial range corresponding to wavenumbers $4 \lesssim k_{\perp} \lesssim 20$. 

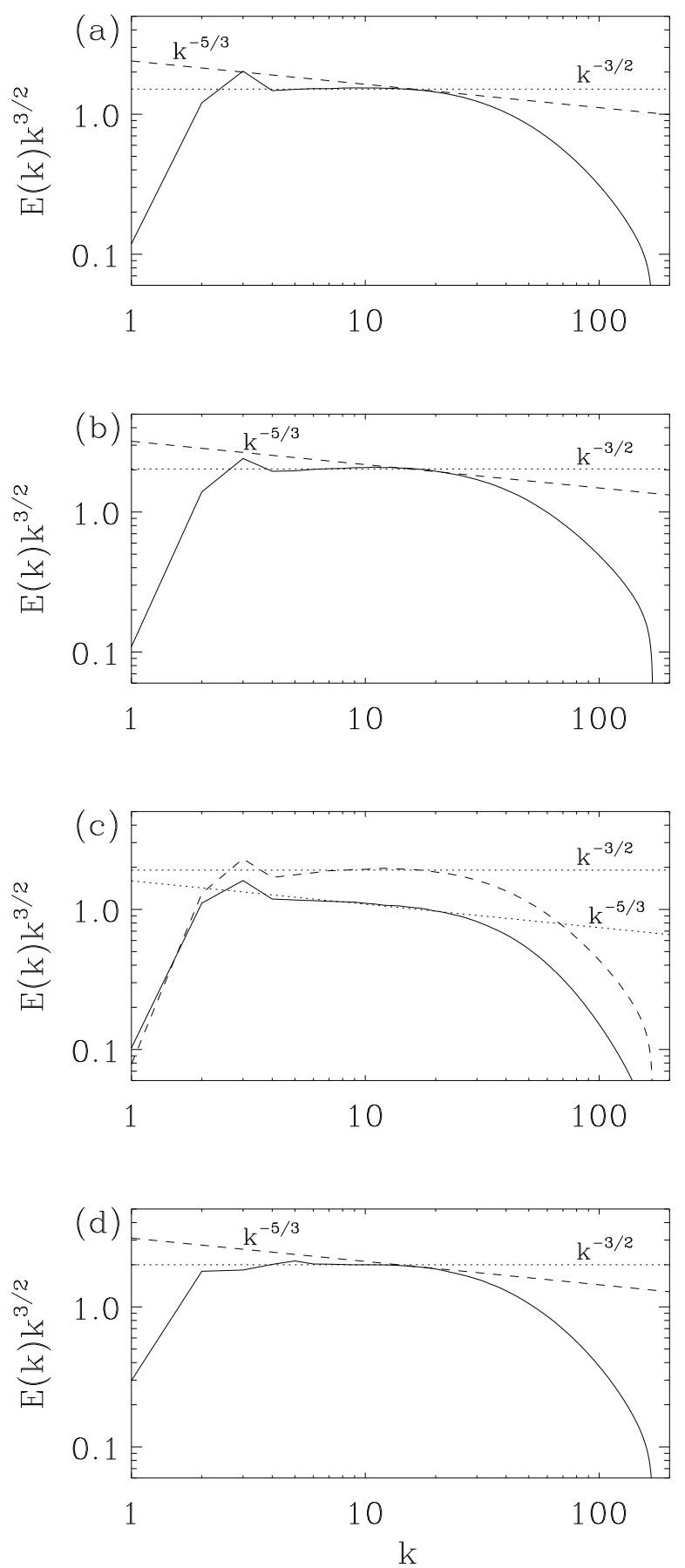

FIG. 1: The (compensated) field-perpendicular energy spectra $E\left(k_{\perp}\right)$ : (a) Case 1; (b) Case 2; (c) Case 1a (solid curve) and 2a (dashed curve); (d) Case 3. The description of the runs is given in Table I.

The corresponding results for Case 2 are illustrated in Figure 1b. Again the exponent $-3 / 2$ is a better fit than $-5 / 3$, with the inertial range corresponding to wavenumbers $4 \lesssim k_{\perp} \lesssim 20$.

In Figure 10 we show the spectra for the above two cases in a special setting when the forcing correlation time is made 10 times shorter. We find that when only
TABLE I: Summary of the results. The forcing correlation time, $\tau$, is given in units of $(L / 2 \pi) / U_{r m s}$.

\begin{tabular}{|c|c|c|c|}
\hline Case & Forcing & Forcing correlation time & Spectrum $E\left(k_{\perp}\right)$ \\
\hline 1 & $\mathrm{u}$ only & 10 & $3 / 2$ \\
\hline 2 & $\mathrm{u}$ and $\mathrm{b}$ & 10 & $3 / 2$ \\
\hline $1 \mathrm{a}$ & $\mathrm{u}$ only & 1 & $5 / 3$ \\
\hline $2 \mathrm{a}$ & $\mathrm{u}$ and $\mathrm{b}$ & 1 & $\lesssim 3 / 2$ \\
\hline 3 & $\mathrm{u}$ and b & frozen large-scale modes & $3 / 2$ \\
\hline
\end{tabular}

the velocity field is forced (Case 1a: solid line) the best fit now appears to be $-5 / 3$ with the inertial range corresponding to wavenumbers $5 \lesssim k_{\perp} \lesssim 23$. When both the magnetic and velocity fields are independently driven (Case 2a: dashed line) the better fitting exponent remains at $-3 / 2$, although the range of wavenumbers over which this holds has become smaller and the fit is worse.

The energy spectrum for Case 3 is shown in Figure 1 d. We note here that we have initialized the simulation with the statistically steady state solution from Case 1a, for which we found the exponent $-5 / 3$. After a certain relaxation time, the energy spectrum of the resulting stationary turbulence is again better fit by $-3 / 2$ with the inertial range corresponding to wavenumbers $4 \lesssim k_{\perp} \lesssim 20$. The scaling exponents found in each case are summarized in Table I. In the next section we offer an explanation for our results.

Finally, we also calculated the alignment angle between the shear-Alfvén velocity and magnetic field fluctuations. It is recalled that the theoretical prediction $\theta \propto \lambda^{1 / 4}$ is thought to be responsible for the field-perpendicular spectrum $E\left(k_{\perp}\right) \propto k_{\perp}^{-3 / 2}[13$, 14, 18, 24]. We define $\delta \mathbf{v}_{r}=\mathbf{v}(\mathbf{x}+\mathbf{r})-\mathbf{v}(\mathbf{x})$ and $\delta \mathbf{b}_{r}=\mathbf{b}(\mathbf{x}+\mathbf{r})-\mathbf{b}(\mathbf{x})$, where $\mathbf{r}$ is a point-separation vector in a plane perpendicular to the large-scale field $\mathbf{B}_{0}$, and for each simulation we measure the ratio (see [18])

$$
\theta_{r} \approx \sin \left(\theta_{r}\right) \equiv \frac{\left\langle\left|\delta \tilde{\mathbf{v}}_{r} \times \delta \tilde{\mathbf{b}}_{r}\right|\right\rangle}{\left\langle\left|\delta \tilde{\mathbf{v}}_{r} \| \delta \tilde{\mathbf{b}}_{r}\right|\right\rangle}
$$

where $\delta \tilde{\mathbf{v}}_{r}=\delta \mathbf{v}_{r}-\left(\delta \mathbf{v}_{r} \cdot \mathbf{n}\right) \mathbf{n}, \delta \tilde{\mathbf{b}}_{r}=\delta \mathbf{b}_{r}-\left(\delta \mathbf{b}_{r} \cdot \mathbf{n}\right) \mathbf{n}$ and $\mathbf{n}=\mathbf{B}(\mathbf{x}) /|\mathbf{B}(\mathbf{x})|$. Figure 2 shows that $\theta \propto \lambda^{1 / 4}$ is good fit for all the simulations. We note that the Goldreich \& Sridhar theory [9] which predicts $E\left(k_{\perp}\right) \propto k_{\perp}^{-5 / 3}$ would be consistent with a zero slope in Figure 2. propose that the reason that the alignment angle is well observed is because its measurement is composed of the ratio of two structure functions, and as such is somewhat analogous to the phenomenon of extended self-similarity [25]. This phenomenon is well known in hydrodynamic turbulence, where a cleaner scaling behavior and longer inertial ranges are apparent when one structure function is considered as a function of the other, rather than considering each separately as functions of $r$. 


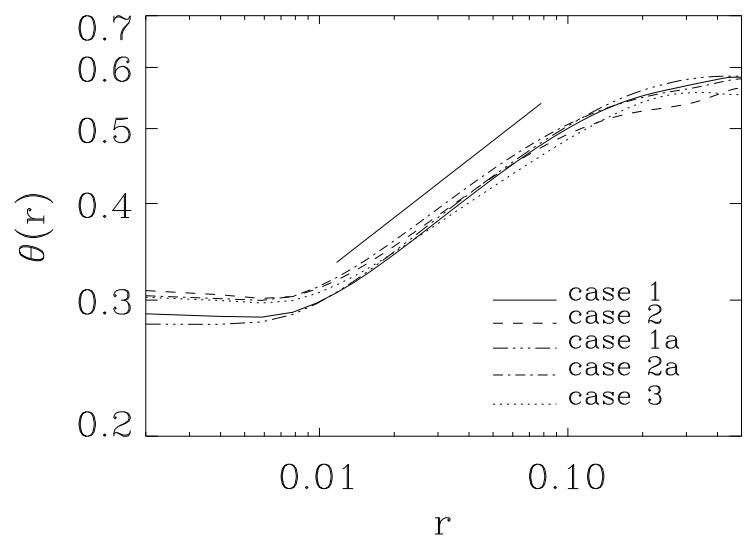

FIG. 2: The alignment angle $\theta$ as a function of scale for each of the simulations. The straight line has slope 0.25 corresponding to the energy spectrum $E\left(k_{\perp}\right) \propto k_{\perp}^{-3 / 2}$, see [13, 14, 18].

Discussion and conclusion.- Our results lead us to believe that the universal spectrum for MHD turbulence in the presence of a strong guiding field is $E\left(k_{\perp}\right) \propto k_{\perp}^{-3 / 2}$. In order to understand the exceptional case (Case 1a) in which we obtain an exponent closer to $-5 / 3$, it is instructive to determine the turnover time of the largescale eddies. This can be done by measuring the energy relaxation time in Case 3, i.e., the characteristic time on which the solution adjusts from a randomly stirred state to a state with frozen large-scale modes. We find that the large-scale eddy turnover time is approximately $\tau_{0} \sim 5$, which agrees with the dimensional estimate $\tau_{0} \approx L / U_{r m s} \approx 2 \pi$.

We then conducted a series of simulations (not shown here) analogous to Case 1a where we varied the force correlation time from $\tau=0.1$ to $\tau=10$. We observed that the transition from the spectrum $E\left(k_{\perp}\right) \propto k_{\perp}^{-5 / 3}$ to $E\left(k_{\perp}\right) \propto k_{\perp}^{-3 / 2}$ occurred between $\tau \approx 1$ and $\tau \approx 2$, which is much smaller than the large-scale eddy turnover time. We believe that a driving mechanism with such a short renovation time is not well suited for investigations of well developed strong MHD turbulence, since an inertial interval of limited extent may be spoiled by the transition region from the forcing interval at scales where the eddy turnover times are larger than the force correlation time. We believe it is this feature that may be responsible for the different exponent in this case. It is reasonable to expect that a larger calculation would yield $E\left(k_{\perp}\right) \propto$ $k_{\perp}^{-3 / 2}$ sufficiently deep in the inertial range, though at the present time such a calculation is not feasible. This issue is currently under investigation by different means, and will be addressed in detail elsewhere.

We thank Jean C. Perez for useful discussions. This work was supported by the NSF Center for Magnetic SelfOrganization in Laboratory and Astrophysical Plasmas at the University of Chicago and the University of Wisconsin at Madison. We appreciate the hospitality and support of the Aspen Center for Physics where a part of this work was performed. We gratefully acknowledge use of "BGL", a 1024-node IBM Blue Gene/L system operated by the Argonne Leadership Computing facility at Argonne National Laboratory.

[1] Biskamp, D., 2003, Magnetohydrodynamic Turbulence. (Cambridge University Press, Cambridge).

[2] Iroshnikov, P. S., AZh, 40 (1963) 742; Sov. Astron, 7 (1964) 566.

[3] Kraichnan, R. H., Phys. Fluids, 8 (1965) 1385.

[4] Kadomtsev, B. B. \& Pogutse, O. P. Sov. Phys.- JETP, 38 (1974) 283.

[5] Strauss, H. R., Phys. Fluids, 19 (1976) 134.

[6] Montgomery, D., Phys. Scripta, T2 (1982) 83.

[7] Shebalin, J. V., Matthaeus, W. H. \& Montgomery, D. J. Plasma Physics, 29 (1983) 525.

[8] Sridhar, S. \& Goldreich, P. ApJ 432 (1994) 612.

[9] Goldreich, P. \& Sridhar, S., ApJ 438 (1995) 763.

[10] Maron, J., \& Goldreich, P., ApJ 554 (2001) 1175.

[11] Müller, W.-C. \& Grappin, R., Phys. Rev. Lett., 95 (2005) 114502.

[12] Müller, W.-C., Biskamp, D., \& Grappin, R., Phys. Rev. E 67 (2003) 066302.

[13] Boldyrev, S., ApJ 626 (2005) L37.

[14] Boldyrev, S., Phys. Rev. Lett. 96 (2006) 115002; astro-ph/0511290

[15] Galtier, S., Pouquet, A., \& Mangeney, A., Phys. Plasmas 12 (2005) 092310.

[16] Chandran, B. D. G., Astrophys. Space Sci. 292 (2004) 17.

[17] Ng, C. S., \& Bhattacharjee, A., ApJ 465 (1996) 845.

[18] Mason, J., Cattaneo, F., \& Boldyrev, S., Phys. Rev. Lett, 97 (2006) 255002; astro-ph/0602382

[19] Müller, W.-C. \& Biskamp, D., Phys. Rev. Lett., 84 (2000) 475.

[20] Haugen, N. E. L., Brandenburg, A., \& Dobler, W., Phys. Rev. E 70 (2004) 016308.

[21] Haugen, N. E. L., Brandenburg, A., \& Dobler, W., Astrophys. Space Sci. 292 (2004) 53.

[22] Cho, J. \& Vishniac, E. T., ApJ. 539 (2000) 273.

[23] Cho, J., Lazarian, A., \& Vishniac, E. T., ApJ. 564 (2002) 291.

[24] Boldyrev, S., Mason, J. \& Cattaneo, F., (2006) arXiv:astro-ph/0605233

[25] Benzi, R., Ciliberto, S., Tripiccione, R., Baudet, C., Massaioli, F., and Succi, S., Phys. Rev. E 48 (1993) R29. 